\title{
Influence of Oxygen Concentration of Si Wafer Surface in Si Emission on Nano Ordered Three-Dimensional Structure Devices
}

\author{
Etsuo Fukuda* and Tetsuo Endoh \\ Center for Innovative Integrated Electronic Systems (cies), Tohoku University, \\ Aoba-ku, Aramaki-aza-aoba, 468-1, Sendai 980-0845, Japan
}

Takashi Ishikawa, Koji Izunome, Kazutaka Kamijo, Moriya Miyashita, and Takao Sakamoto
Global Wafers Japan, Kitakanbara-gun, Seiro-cho, Higashikou, 6-861, Niigata 957-0101, Japan

\section{Hiroyuki Kageshima}

Department of Mechanical, Electrical and Electronic Engineering, Interdisciplinary Gradate Science and Engineering, Shimane University, Matsue-shi, Nishikawatsu-machi, 1060, Shimane 690-8504, Japan

(Received 23 October 2017; Accepted 15 November 2017; Published 14 December 2017)

\begin{abstract}
In past studies, the Si emission phenomenon is one of the issues for fabrication of 3D structure devices such as FinFETs and Vertical MOSFETs. In this paper, it is found that novel Si emission phenomena depending on the surface oxygen concentration of $\mathrm{Si}$ wafer occur, when Si pillars patterned less than $100 \mathrm{~nm}$ are oxidized. A wafer with high oxygen concentration which is over $1.0 \times 10^{18}$ atoms $/ \mathrm{cm}^{3}$ can suppress Si emission from the $\mathrm{Si}$ pillar compared to the low oxygen concentration wafers which are less than $1.0 \times 10^{17}$ atoms $/ \mathrm{cm}^{3}$. The difference of oxygen concentration in the Si substrate is expected to largely depend on the behavior of oxygen atom in the Si wafer before and after oxidation. In case of an oxygen concentration ratio exceeding the solid solubility of $\mathrm{Si}$, oxygen diffuses outward from the Si substrate after oxidation, whereas oxygen diffuses inward when the concentration is below the solid solubility. It was also found that the larger the degree of injection of oxygen into the Si substrate after oxidation, the larger the emission amount of Si from the Si pillar. Finally, we discuss the mechanism of above experimental Si emission phenomena in nanoscale Si pillar with previous first principle model of silicon oxidation process. [DOI: 10.1380/ejssnt.2017.127]
\end{abstract}

Keywords: Oxygen; Oxidation; Silicon oxides; Single crystal surfaces; Surface roughness; Semiconductor-insulator interfaces; $\operatorname{Si}(100)$

\section{INTRODUCTION}

As one means for solving the limitation of miniaturization of the Si devices, a device structure called multigate transistor [1] which is much different from the conventional planar type has been devised. In this threedimensional transistor, a three-dimensional structured device called "Tri Gate Transistor [2-5]" which has gates on three planes so as to sandwich the channel, has been proposed. In particular, Body Channel type vertical MOSFET (BC-MOSFET: generally called SGT (Surrounding Gate Transistor [6-11])) is characterized in that the source, gate and drain electrodes are vertically arranged and have a $\mathrm{Si}$ pillar shape. In the manufacturing process of the BC-MOSFET, there is a step of oxidizing its surface for forming a gate oxide film after forming the $\mathrm{Si}$ Pillar. It is necessary to narrow the diameter of the Si Pillar for higher density integration. In the case of a pillar diameter of several tens of nm or less, when high temperature heat is applied by thermal oxidation process, silicon atoms constituting the pillar crystal moves and the phenomenon occurs in which the pillar structure cannot be maintained. Such thermal oxidation of the Si pillar structure already has been studied by many researchers [1219]. Liu et.al. [15-17] has reported that $\mathrm{Si}$ atoms in the $\mathrm{Si}$ pillar disappear after thermal oxidation with dry $\mathrm{O}_{2}$, at $850^{\circ} \mathrm{C}$ for 5 hours. According to Kageshima etal., the $\mathrm{Si}$ emission is a major cause for the missing $\mathrm{Si}$ [20-29]. It is

\footnotetext{
* Corresponding author: efukuda@cies.tohoku.ac.jp
}

also reported that the amount of $\mathrm{Si}$ emission is larger in the pillar-shape Si oxidation than in the planar Si oxidation, where the emission becomes more prominent as the Si pillar diameter becomes smaller. Since the Si pillar is the part that conducts the current in the BC-MOSFET, if the Si pillar narrows or breaks by missing $\mathrm{Si}$, the $\mathrm{BC}$ MOSFET cannot maintain performance as a transistor. As a result, the missing $\mathrm{Si}$ causes a great decrease in yield and reliability of the transistors.

Regarding the Si emission in thermal oxidation as mentioned above, although a great deal of research has been carried out, neither of them takes the influence of impurities such as oxygen in the $\mathrm{Si}$ wafer into consideration. In this paper, the influence of the oxygen concentration on the surface of Si substrate forming the nano-order $\mathrm{Si}$ pillar with the phenomenon of Si missing is reported.

\section{EXPERIMENTAL}

\section{A. Hypothesis of the Si emission}

In recent device structures, many kinds of stresses are generated in the Si substrate due to the influence of deposition of different permittivity films and complex device shape. For this reason, this stress distorts the Si substrate, forming dislocations, stacking faults and so on. These formed dislocations and defects are sources of leakage current which deteriorates transistor performances (Fig. 1(a) [30]). In the pillar-shape MOSFET of this paper as well, similar stresses are applied to the pillar structure, and as a result, it was predicted that dislocations 


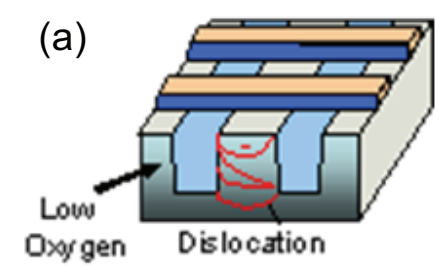

(b)

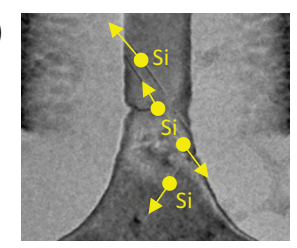

FIG. 1. Hypothesis of Si emission for (a) Dislocation due to structural stress in the device, (b) Image of $\mathrm{Si}$ emission via dislocation in the Si pillar.

and stacking faults occur inside the pillar. Then, the Si missing hypothesizes that silicon may be missioned inside or outside the Si substrate by this generated dislocation.

As a method described above for suppressing dislocation in the Si wafer surface layer, it is known to utilize the fixing effect of oxygen [31-35]. It is known that the $\mathrm{Si}$ substrate of the CZ method with higher oxygen concentration suppresses the shear stress than the Si substrate of the FZ (Floating Zone) method with less oxygen in the Si substrate. This phenomenon is also called the pinning effect [36] because oxygen in the Si substrate pinned the dislocation. Therefore, we formulate a hypothesis that, if the cause of $\mathrm{Si}$ emission in the $\mathrm{Si}$ pillar structure is attributed to dislocations and defects due to substrate stress, increasing the oxygen concentration in the surface layer of the Si wafer can suppress the Si emission.

From the conclusion of this hypothesis at this point, it will be clarified by TEM observation of the Si pillar in the later chapter, but dislocations and stacking faults could not be visualized from the change in shape Si pillar used in the experiment. Therefore, at the first stage, it was only shown that the cause of the Si missing was not caused by dislocations or defects. Furthermore, the following experiments have shown that the oxygen concentration in the $\mathrm{Si}$ substrate selected as one of the experimental parameters has a large effect on the Si missing phenomena.

\section{B. The oxygen concentration of the Si substrate sample used for the experiment}

Two types of Si wafers A and C with different oxygen concentrations were prepared for experiments. Two types of oxygen concentration distributions obtained by SIMS analysis are shown in Fig. 2. Figure 2 expresses the relation between the oxygen concentration on the vertical axis and the depth from the Si substrate surface on the horizontal axis. Sample A has an oxygen concentration of about $1.0 \times 10^{18}$ atoms $/ \mathrm{cm}^{3}$ at the surface layer, where sample $\mathrm{C}$ is around $1.0 \times 10^{16}$ atoms $/ \mathrm{cm}^{3}$. By using these two types of $\mathrm{Si}$ wafers, experiments were conducted on the effect of the Si missing by oxidizing the Nano-order Si pillar.

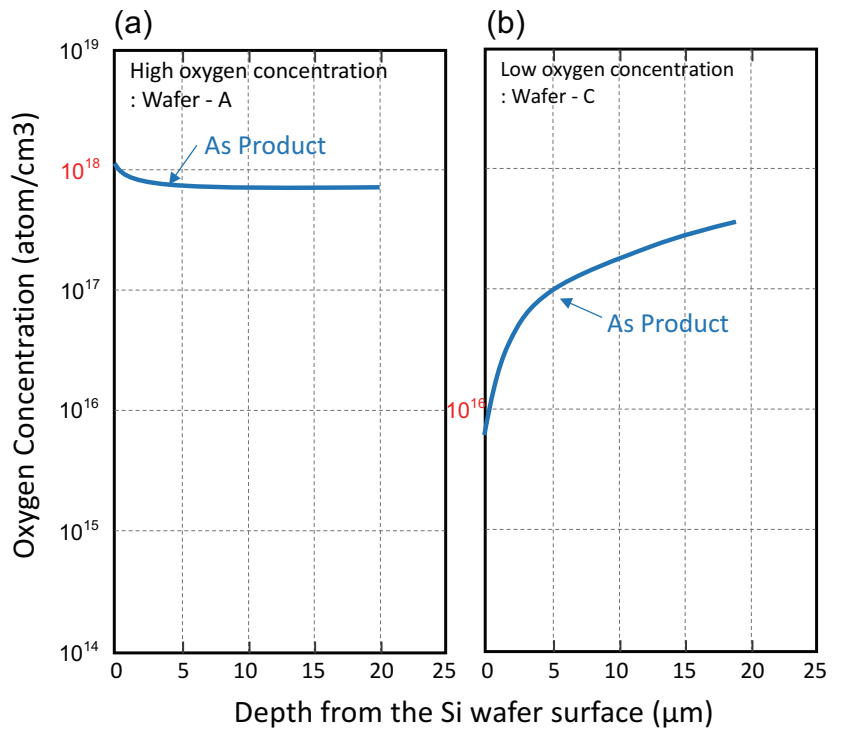

FIG. 2. Oxygen Concentration in Si wafer substrate of two samples for (a) High oxygen concentration: Wafer-A, (b) Low oxygen concentration: Wafer-C.

(a)

(b)

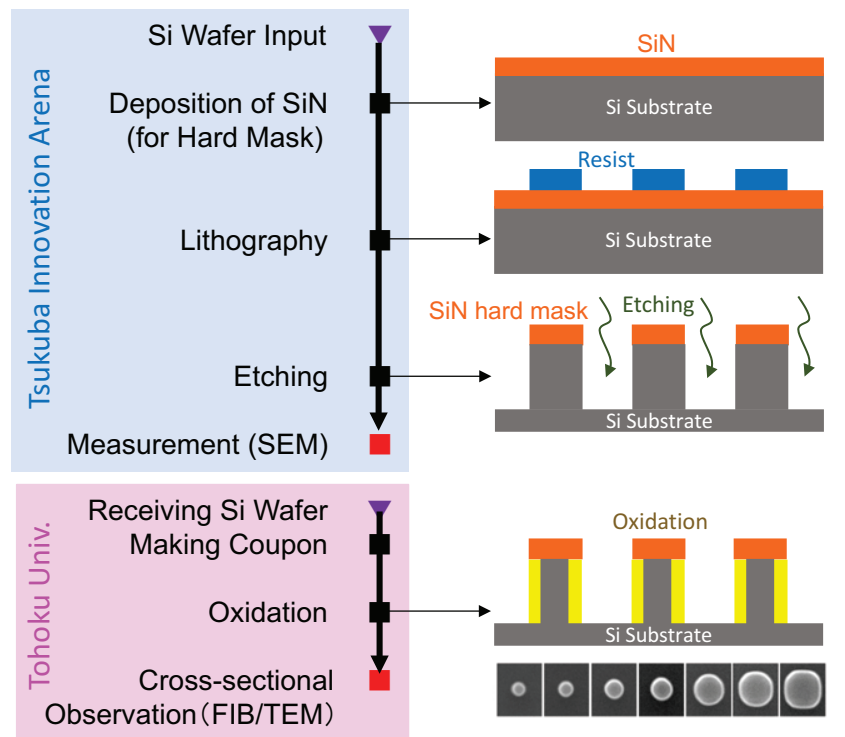

FIG. 3. Process Flow for the Si Nano pillar formation for (a) Process Flow, (b) Image of Process cross section.

\section{Si pillar manufacturing process flow}

The Si pillar formation process flow is shown in Fig. 3. This is a simple process flow to produce the Si pillar on a Si wafer using lithography and etching process with SiN film as a hard mask. The lithography process was carried out using the Tsukuba Innovation Arena (TIA) at Tsukuba Industrial Research Institute. Oxidation process after $\mathrm{Si}$ etching was performed using an oxidation furnace in Tohoku University after shaping Si Wafer returned from TIA into a coupon. 
TABLE I. Effect of precipitating agent on BET surface area of $\mathrm{CuO}$

\begin{tabular}{cccc}
\hline \hline & Condition 1 & Condition 2 & Condition 3 \\
\hline Si pillar diameter & $70 \mathrm{~nm}$ & $90 \mathrm{~nm}$ & $100 \mathrm{~nm}$ \\
Oxidation Thickness on $\mathrm{Si}(100)$ & $20 \mathrm{~nm}$ & $30 \mathrm{~nm}$ & $40 \mathrm{~nm}$ \\
Oxidation Temperature $\left(\right.$ dry $\left.\mathrm{O}_{2}\right)$ & $800^{\circ} \mathrm{C}$ & $900^{\circ} \mathrm{C}$ & $1000^{\circ} \mathrm{C}$ \\
\hline \hline
\end{tabular}

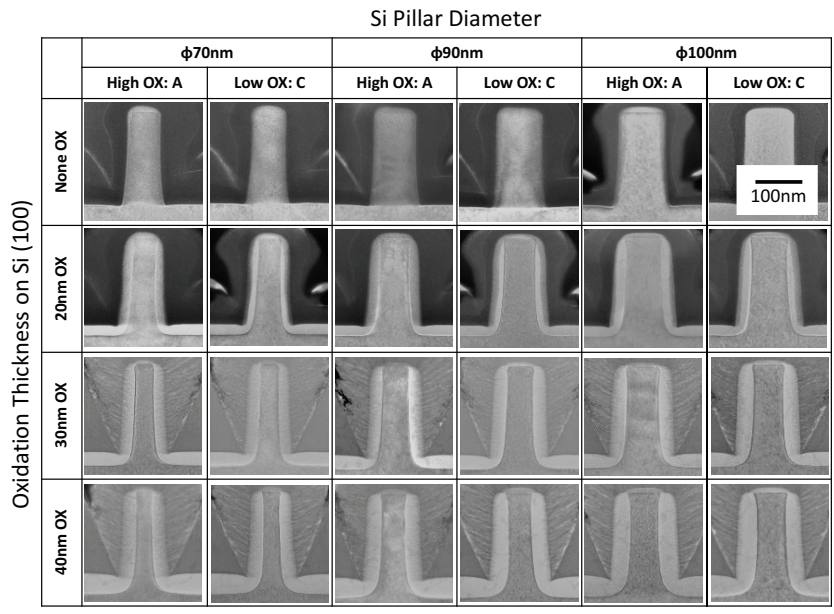

FIG. 4. Oxidation Thickness dependency on Si pillar diameter (Si pillar Cross-sectional Photography by TEM).

\section{Experiment parameter list}

Three types of the Si pillar diameters of $70 \mathrm{~nm}, 90 \mathrm{~nm}$, and $100 \mathrm{~nm}$ were prepared as the samples. Oxidation was performed by dry $\mathrm{O}_{2}$ oxygen, and the aimed oxide film $\left(\mathrm{SiO}_{2}\right)$ thickness was $20 \mathrm{~nm}, 30 \mathrm{~nm}$, and $40 \mathrm{~nm}$ on the $\mathrm{Si}(100)$ plane. The oxidation temperature was set to three kinds of temperatures, $800^{\circ} \mathrm{C}, 900^{\circ} \mathrm{C}$ and $1000^{\circ} \mathrm{C}$, respectively. These parameters are summarized in Table I.

\section{RESULTS AND DISCUSSIONS}

\section{A. Dependence of Si pillar diameter and oxide film thickness}

At first, experiments on the dependence of Si pillar diameter and oxide thickness were carried out using two types of wafer A and C (Fig. 4). The oxide film thickness was conditioned as $20 \mathrm{~nm}, 30 \mathrm{~nm}$ and $40 \mathrm{~nm}$, respectively, maintaining oxidation temperature 900 with dry $\mathrm{O}_{2}$ under 1 atom (Is the unit correct?). The Si pillar diameter was $70 \mathrm{~nm}, 90 \mathrm{~nm}, 100 \mathrm{~nm}$, respectively.

The experimental results are shown in Fig. 4. This figure is a summary of the photographs of Si pillars after processing with FIB and observing these cross sections with TEM. For wafers A and C, respectively, the vertical axis represents oxide film thicknesses, and the horizontal axis expresses Si pillar diameter. (a)

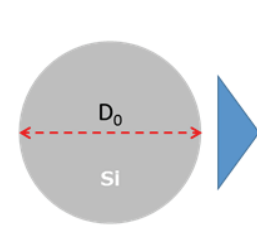

Before Oxidation (b)

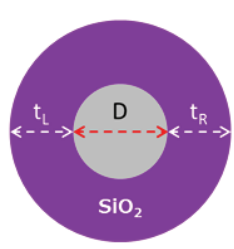

After Oxidation

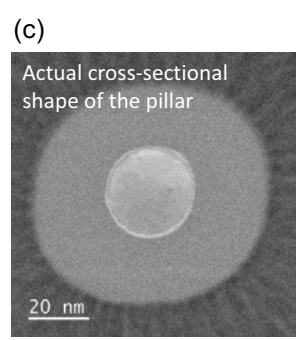

FIG. 5. XRD patterns of pure and binary metal oxide nanoparticles

\section{B. Calculation method of $\mathrm{Si}$ atom reduction amount}

The Si pillar diameter of the vertical section TEM image shown in Fig. 4 was measured, and the change in the $\mathrm{Si}$ atom amount of Si pillar body was calculated. Although there are some differences in the horizontal cross section shape among the observed samples, assuming that all Si pillar sections are circular, the calculation of the Si pillar volume was calculated as a cylinder (Fig. 5). Changes in the number of Si atoms before and after oxidation were calculated. The calculation formula is shown in (1) $\left(\mathrm{N}_{\mathrm{Si}}=51\right.$ atoms $/ \mathrm{nm}^{3}$ and $\mathrm{N}_{\mathrm{SiO}_{2}}=20$ atoms $/ \mathrm{nm}^{3}$ are used).

$$
\begin{aligned}
f(x) & \text { : Si Residual ratio } \\
& =\frac{N_{\mathrm{Si}} \pi\left(\frac{D}{2}\right)^{2}+N_{\mathrm{SiO}_{2}} \pi\left[\left(\frac{t_{L}+D+t_{R}}{2}\right)^{2}-\left(\frac{D}{2}\right)^{2}\right]}{N_{\mathrm{Si}} \pi\left(\frac{D}{2}\right)^{2}}
\end{aligned}
$$

\section{Consideration on difference of Si missing amount depending on the Si pillar diameter and oxide film thickness dependence}

The residual rate of Si atoms is visualized in Fig. 6 using size of circle area. The Si pillar diameter is plotted on the vertical axis and oxide film thickness is expressed on the horizontal axis, and the amount of Si missing is graphically represented by the size of the circle. The numerical value inside the circle indicates the ratio (\%) of Si missing, and it is shown that the larger the area of the circle, the larger the amount of Si missing in Fig. 6. As mentioned before, however, there are variations in the shape of the Si pillars, so there is no quantitative accuracy in the numerical value of the ratio of Si missing. Therefore, the size of the circle represents the amount of Si missing as a qualitative or relative difference. 


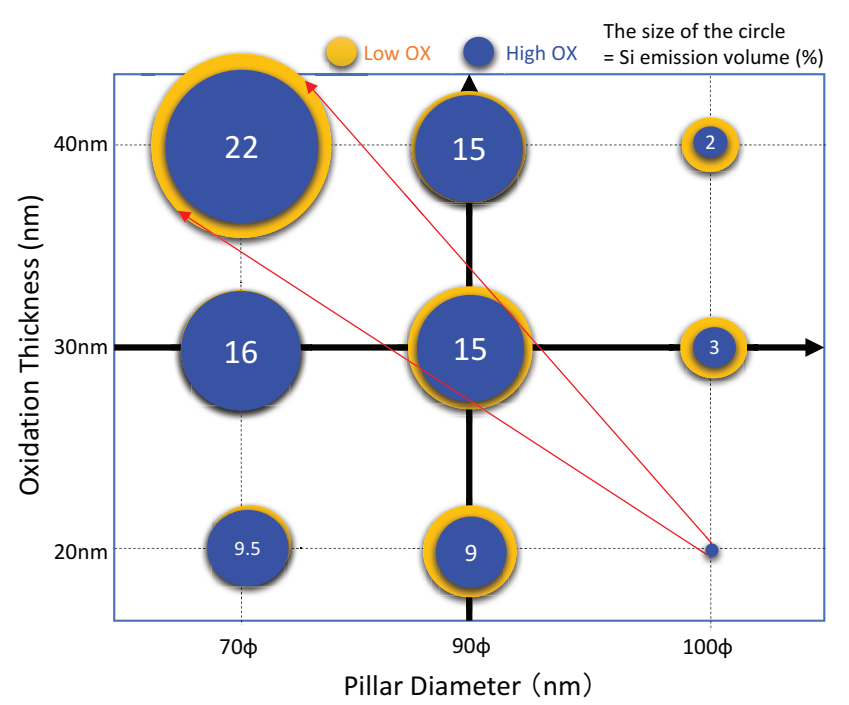

FIG. 6. Dependence between Oxidation thickness and the Si pillar Diameter for Si atoms missing volume (The circle size corresponds to Si missing volume) by $900^{\circ} \mathrm{C}$ oxidation. Yellow circle shows low oxygen wafer, blue one shows high oxygen wafer.

It was found that the smaller the Si pillar diameter and the thicker the oxide film, the higher the amount of Si missing qualitatively. Comparing the two wafers of high oxygen $\mathrm{A}$ and low oxygen $\mathrm{C}$, it was also found that the wafer A of high oxygen concentration suppresses the amount of Si missing relatively. Summary of the dependence results of both the Si pillar diameter and oxide thickness is as follows;

1. The Si missing is more prominent as the Si pillar diameter is smaller and the oxide film is thicker.

2. The high oxygen wafer A tends to have less Si missing compared to the low oxygen wafer $\mathrm{C}$.

3. The shape dependence that leads to different Si missing amount exists depending on the thickness and shape of the Si pillar.

\section{Difference of Si missing amount depending on the Si pillar diameter and oxidation temperature dependence}

In this section, the dependence between pillar diameter and oxidation temperature of the experiment is examined. With the oxide film thickness fixed at $40 \mathrm{~nm}$, oxidation temperatures are set to three conditions of $800^{\circ} \mathrm{C}, 900^{\circ} \mathrm{C}$, and $1000^{\circ} \mathrm{C}$, respectively. These parameters are summarized in Table II.

The residual Si atom ratio was calculated by the same method as in Section III.B and summarized in Fig. 7. The expression method of the graph is also the same as in Section III.C. The amount of Si atom emission depends mostly on the pillar diameter. With the smaller diameter, obviously, the Si missing amount tends to increase as shown in Fig. 7. The dependence of the oxidation temperature on the Si pillar diameter is different. At $70 \mathrm{~nm}$

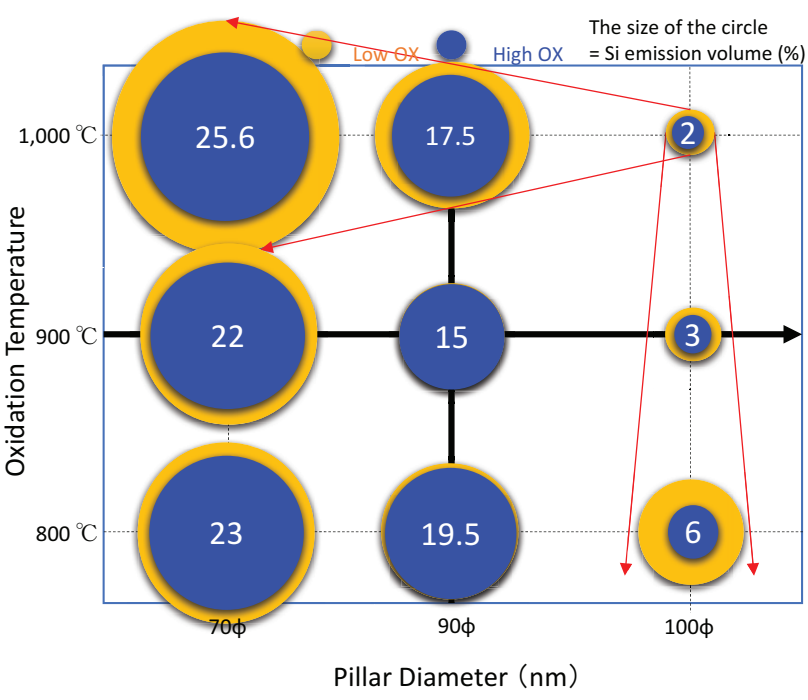

FIG. 7. Dependence of between oxidation thickness and the Si pillar diameter for Si atoms missing volume (The circle size corresponds to emission volume) on $40 \mathrm{~nm}$ oxidation. Yellow circle shows low oxygen wafer, blue one shows high oxygen wafer.

with a small Pillar diameter, Si missing at temperature of $1000^{\circ} \mathrm{C}$ is remarkable, whereas at a sample with a $100 \mathrm{~nm}$ thick Si pillar diameter, the Si missing amount tends to be larger than $1000^{\circ} \mathrm{C}$ at a low temperature of $800^{\circ} \mathrm{C}$. As a difference between the two kinds of wafers, it can be seen that the low oxygen wafers $\mathrm{C}$ generally releases more Si atoms. Summary of the dependence results of both the Si pillar diameter and oxidation temperature is given as follows;

1. The Si missing is prominent as the Si pillar diameter is smaller and the oxidation temperature is higher (higher energy).

2. Also, the difference due to the oxygen concentration of Si wafer also remarkably appears. A wafer with lower oxygen concentration emits more silicon.

3. In a thick $100 \mathrm{~nm}$ Si pillar low oxygen wafer, the lower the temperature the more the Si missing.

\section{ANALYSIS USING TEM}

In order to further analyze the difference between high oxygen wafer $\mathrm{A}$ and low oxygen wafer $\mathrm{C}$, detailed observation was carried out using high resolution BF-STEM [37]. When the Si pillar sample was made with a pillar diameter of $70 \mathrm{~nm}$, oxide thickness of $40 \mathrm{~nm}$, temperature of $900^{\circ} \mathrm{C}$, dry $\mathrm{O}_{2}$ oxidation, the most prominent Si missing was observed.

\section{A. Difference of $\mathrm{Si} / \mathrm{SiO}_{2}$ interface Roughness}

Figure 8 (a) shows the Si pillar shape of high and low oxygen concentration, respectively before oxidation. Fig- 
TABLE II. BET surface area and average crystal size of single and binary oxide nanoparticles

\begin{tabular}{cccc}
\hline \hline & Condition 1 & Condition 2 & Condition 3 \\
\hline Pillar diameter & $70 \mathrm{~nm}$ & $90 \mathrm{~nm}$ & $100 \mathrm{~nm}$ \\
Oxidation Thickness on $\operatorname{Si}(100)$ & & & $40 \mathrm{~nm}$ \\
Oxidation Temperature $\left(\right.$ dry $\left.\mathrm{O}_{2}\right)$ & $800^{\circ} \mathrm{C}$ & $900^{\circ} \mathrm{C}$ & $1000^{\circ} \mathrm{C}$ \\
Oxidation Pressure & & 1 atom & \\
\hline \hline
\end{tabular}

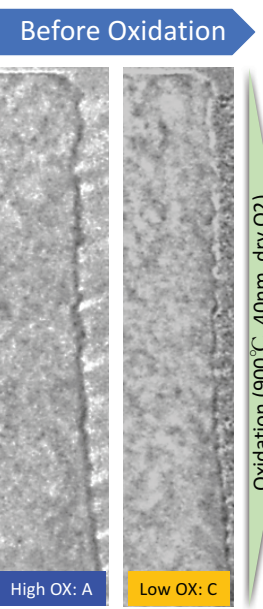

(2) Before Oxidation

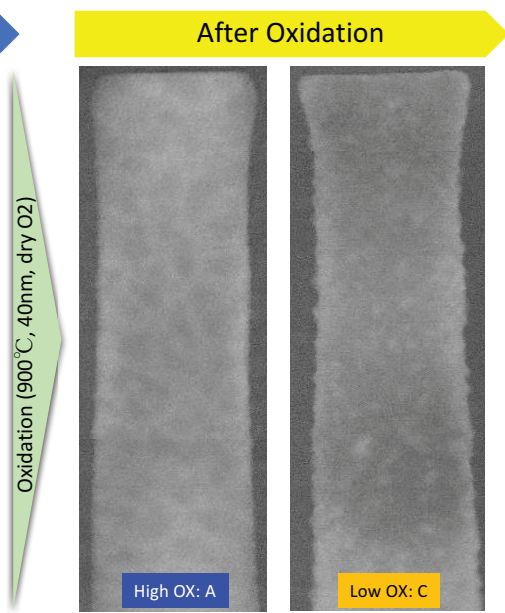

(b) After Oxidation
FIG. 8. BF-STEM image comparison of oxidized sample with the Si pillar diameter $70 \mathrm{~nm}$, oxidation thickness 40 for (a) before oxidation, (b) after oxidation.

ure $8(\mathrm{~b})$ is a picture of each Si pillar shapes after oxidation. Before oxidation, there is no big difference in the surface roughness of each Si pillar side, and it turns out that it is uneven shape. It is observed that the pillar surfaces are uneven with both pillars before oxidation. However, it is turned out that there is a big difference in the roughness of the $\mathrm{Si} / \mathrm{SiO}_{2}$ interface for both, after oxidation of $40 \mathrm{~nm}$. Obviously the pillars of high oxygen Si wafer A has a smooth surface shape with no irregularities compared to the pillars of low oxygen wafer C.

\section{B. High resolution observation of $\mathrm{Si} / \mathrm{SiO}_{2}$ interface roughness}

The results of observing the details of the $\mathrm{Si}$ pillar by raising the resolution further are shown in this section. Comparative photographs of two types of wafers which are high and low oxygen concentration wafers at Pillar's footpath, Pillar's head corner, Si(100) surface position, respectively are shown in Fig. 9. These observations clearly show that the high oxygen concentration wafer A clearly distinguishes the $\mathrm{Si} / \mathrm{SiO}_{2}$ interface which boundary is clear instead of the low oxygen concentration wafer $\mathrm{C}$. In other words, the difference in uneven roughness seems to be due to the clarity of this $\mathrm{Si} / \mathrm{SiO}_{2}$ interface. However, as shown in Fig. 9 (c), no difference in roughness of the $\mathrm{Si} / \mathrm{SiO}_{2}$ interface is observed on the flat surface of $\mathrm{Si}(100)$. The difference in the $\mathrm{Si} / \mathrm{SiO}_{2}$ interfacial roughness depending on the oxygen concentration (a) at Pillar's footpath

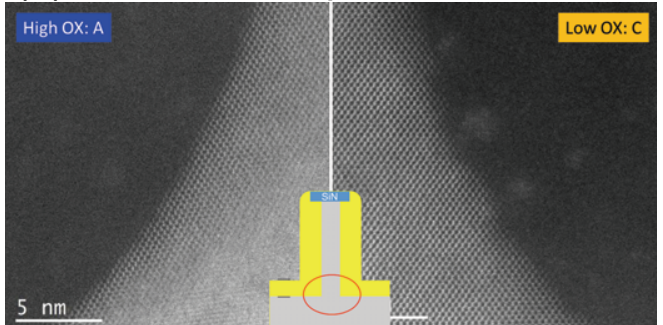

(b) at Pillar's head corner

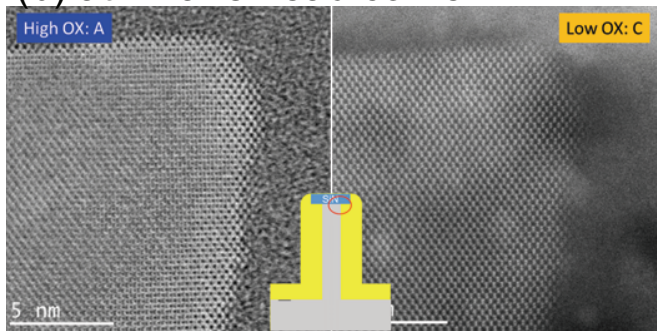

(c) on Si (100) surface

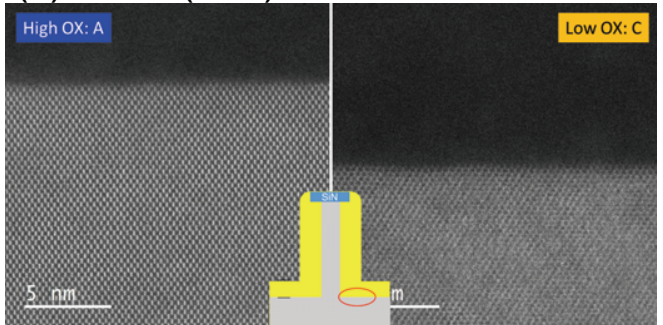

FIG. 9. Comparison of high resolution STEM image for (a) at pillar's footpath, (b) at pillar's head corner, (c) on $\operatorname{Si}(100)$ surface.

in the Si substrate is a phenomenon found in the nanoorder three-dimension shape like the Si pillar shown in photographs of Fig. 9.

\section{Sub conclusion of TEM Analysis}

The results of TEM analysis are summarized in Table III.

Toray Research has analyzed the $\mathrm{Si} / \mathrm{SiO}_{2}$ interface in detail [38]. According to that report, a large amount of oxygen is diffused in the surface layer on the Si substrate side of the $\mathrm{Si} / \mathrm{SiO}_{2}$ interface. Due to the existence of oxygen atoms which entered between the Si lattices, the interface boundary seems to be black and unclear. In other words, the difference in the roughness of the $\mathrm{Si} / \mathrm{SiO}_{2}$ interface is considered to be difficult to judge the interface 
TABLE III. BET surface area and average crystal size of single and binary oxide nanoparticles

\begin{tabular}{lccc}
\hline \hline Wafer Type & Amount of Si & \multicolumn{2}{c}{ Roughness on Si/SiO 2 interface } \\
\cline { 3 - 3 } & emission & Dislocation/ & After OX \\
Low Oxygen & High emission & Worse/uneven & Worse/uneven \\
High Oxygen & Low emission & Norse & Smooth \\
\hline \hline
\end{tabular}
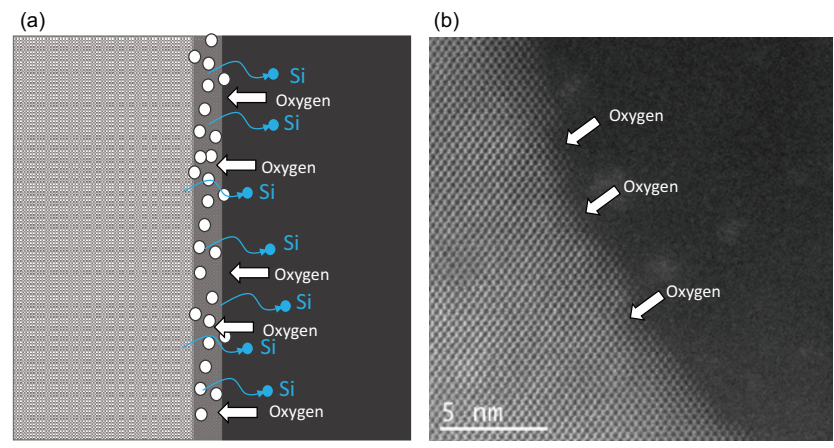

FIG. 10. $\mathrm{Si} / \mathrm{SiO}_{2}$ interface for (a) Image where oxygen is injected into Si substrate and (b) Actual TEM Photography.

boundary due to the fact that oxygen atoms diffuse a lot in the surface layer of the interface on the Si substrate side. And the difference in the amount of oxygen entering the Si substrate is the presumable cause of the difference in the amount of Si missing (Fig. 9).

\section{Oxygen concentration change in Si Wafer surface layer after Oxidation}

The next point of focus is the oxygen concentration change before and after oxidation of the Si wafer surface layer. In the high oxygen wafer $\mathrm{A}$ and the low oxygen wafer $\mathrm{C}$, the difference in the oxygen concentration in the Si surface layer was observed. The measurement results by SIMS are shown in Fig. 11. In the same figure, the temperature dependency of the solid solubility of oxygen in the Si crystal is also shown. The oxygen concentration in the surface layer of $\mathrm{Si}$ wafer before oxidation (blue line) and after oxidation at $900^{\circ} \mathrm{C}$ for 4 hours (red line) was measured. It is clear from this experiment that high oxygen wafer $\mathrm{A}$ and low oxygen wafer $\mathrm{C}$ have a large difference in oxygen concentration change in the Si surface layer. In the high oxygen wafer $\mathrm{A}$, the oxygen concentration in the surface layer decreases by almost one order of magnitude after oxidation (oxygen diffuses outward from the Si surface), whereas in the low oxygen wafer $\mathrm{C}$, the oxygen concentration is found to be higher by one order or more after oxidation on the contrary. Considering that the solid solubility of oxygen at $900^{\circ} \mathrm{C}$ is $1.0 \times 10^{17}$ atoms $/ \mathrm{cm}^{3}$, this experimental fact is considered as follows;

In the wafer A in which oxygen is contained in the solid solubility or higher, oxygen is diffused to the outside of Si surface by heat treatment. On the other hand, in the low oxygen wafers $\mathrm{C}$ below the solid solubility, the oxygen in the Si substrate is deficient and oxygen is diffused

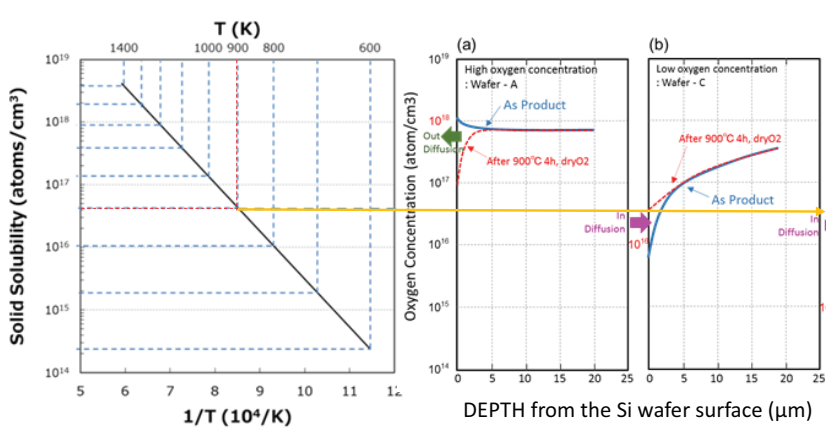

FIG. 11. Change of oxygen concentration after oxidation $\left(900^{\circ} \mathrm{C}, 4 \mathrm{~h}\right.$, dry $\left.\mathrm{O}_{2}\right)$ with Solid solubility in Si crystal. (a) High concentration wafer: A, (b) Low concentration wafer: C.

inwardly into the Si substrate.

From this experimental fact, it is suggested that the larger the amount of oxygen entering the Si substrate, the smaller the amount of Si missing. This interpretation naturally explains that the wafer $\mathrm{A}$, where the oxygen diffuses from the Si surface to outside, can suppress the Si missing.

\section{RESULT AND DISCUSSIONS}

\section{A. Confirmation of Si missing phenomenon}

In the three dimensional Si pillar shape of Nano order, the Si missing phenomenon was confirmed after oxidation. The amount of Si missing depends on the length of the pillar diameter, oxidation time and temperature. It was found that the Si pillar shape is thin with dry $\mathrm{O}_{2}$ oxidation at $900^{\circ} \mathrm{C}$, and more $\mathrm{Si}$ atoms is emitted as the oxidation time is longer. In experiments with constant oxidation time and oxidation temperature, the Si missing was more pronounced as the Si pillar shape is thinner and the temperature is higher. On the other hand, depending on the Si pillar shape, it was found that the amount of the Si missing becomes higher with the lower temperature condition. It was also found that there is no oxygen concentration dependence of $\mathrm{Si}$ wafer depending on the shape. This result confirms that the amount of the missing $\mathrm{Si}$ is enhanced by the geometrical effect of the pillar structure. This is consistent with the Kageshima etal., theory stating that the $\mathrm{Si}$ emission is a major cause for the missing $\mathrm{Si}$, because they predicted that the Si emission is also enhanced by the geometrical effect of the pillar structure [20]. This also suggests that the missing $\mathrm{Si}$ is not easy to be avoided for thin pillar structures. 


\section{B. Difference in Si missing amount due to oxygen} concentration difference in $\mathrm{Si}$ wafer substrate

A large difference in the amount of Si missing was found due to the difference in oxygen concentration in the $\mathrm{Si}$ wafer substrate. The Si wafer containing an oxygen concentration of $1.0 \times 10^{18}$ atoms $/ \mathrm{cm}^{3}$ or more surely suppresses Si missing compared to the wafer, which has lower oxygen concentration than that. Thus, the Si wafer with high oxygen concentration is effective in developing the next generation Nano-order transistors.

The difference in oxygen concentration of the Si wafer is closely related to the solid solubility of oxygen. Since concentration of $1.0 \times 10^{18}$ atoms $/ \mathrm{cm}^{3}$ is higher than the solid solubility in $\mathrm{Si}$ wafer at an oxidation temperature of $900^{\circ} \mathrm{C}$ after oxidation, oxygen diffuses outward from the Si wafer substrate and is emitted outside the Si substrate. On the other hand, since the oxygen concentration is lower than the solid solubility in the Si wafer containing an oxygen concentration of $1.0 \times 10^{16}$ atoms $/ \mathrm{cm}^{3}$ in the Si substrate, oxygen in the Si substrate is in short supply, and oxygen is inwardly diffused into the Si substrate by oxidation. These outward and inward diffusion of oxygen is closely related to the amount of Si missing.

Considering that the $\mathrm{Si}$ missing is the result of the $\mathrm{Si}$ emission, this also means that the Si emission may certainly be controlled by the oxygen concentration difference in Si wafer substrate. Table III indicates that the concentration of self-interstitial is not so high, neither for the Wafer A nor for the Wafer C. This clearly means that the self-interstitial is not the major cause of missing $\mathrm{Si}$. This also suggests that the emitted Si does not necessarily become self-interstitial. Alternatively, the emitted Si must flows into the oxide, and causes the missing Si. Fig- ure 1 and Table III also suggest that the higher oxygen concentration of wafer can reduce the Si emission. This might be the reason why the Si missing is controlled by the difference of the Wafer A and Wafer C.

\section{CONCLUSIONS}

We found that there is a difference in the amount of the Si emission in the Nano order three-dimensional pillar shape due to the difference in oxygen concentration in the Si substrate. It was found that the Si substrate containing more oxygen than the oxygen solid solubility of the Si substrate suppressed the Si emission. Therefore, just using Si substrate with high oxygen concentration is thought to contribute greatly in terms of manufacturing cost of future 3D devices without installing special equipments that suppresses Si emission. In the future, we will pursue the principle as to whether oxygen outwardly diffusing from the Si substrate suppresses Si emission and the principle as to whether oxygen diffusing inward promotes Si emission.

\section{ACKNOWLEDGMENTS}

In STEM analysis, we would like to thank you Professor Konno and Professor Nishijima Metal Materials Research Institute Tohoku University for taking care of us. This work was supported by ACCEL project, "ThreeDimensional Integrated Circuits Technology Based on Vertical BC-MOSFETs and Its Advanced Application Exploration (PL: Tetsuo Endoh, PM: Toru Masaoka)" under Japan Science and Technology Agency.
[1] I. Ferain, C. A. Colinge, and J.-P. Colinge, Nature 479, 310 (2011).

[2] B. S. Doyle, S. Datta, M. Doczy, S. Hareland, B. Jin, J. Kavalieros, T. Linton, A. Murthy, R. Rios, and R. Chau: IEEE Electron Dev. Lett. 24, 263 (2003).

[3] M. Bohr and K. Mistry, Intel's revolutionary $22 \mathrm{~nm}$ transistor technology, Intel website (2011).

[4] D. Hisamoto, T. Kaga, Y. Kawamoto, and E. Takeda, International Technical Digest on Electron Devices Meeting, 833 (1989)

[5] N. Lindert, L. Chang, Y.-K. Choi, E. H. Anderson, W.-C. Lee, T.-J. King, J. Bokor, and C. Hu, IEEE Electron Dev. Lett. 22, 487 (2001).

[6] T. Endoh, T. Nakamura, and F. Masuoka, IEICE Trans. Electron. E80-C, 911 (1997).

[7] T. Endoh, M. Suzuki, H. Sakuraba, and F. Masuoka, IEEE Trans. Electron Devices 48, 1599 (2001).

[8] T. Endoh, K. Kinoshita, T. Tanigami, Y. Wada, K. Sato, K. Yamada, T. Yokoyama, N. Takeuchi, K. Tanaka, N. Awaya, K. Sakiyama, and F. Masuoka, IEEE Trans. Electron Devices 50, 945 (2003).

[9] T. Endoh, K. Sakui, and Y. Yasuda, IEICE Trans. Electron. E93-C, 557 (2010).

[10] T. Endoh and K. Tanaka, Jpn. J. Appl. Phys. 46, 3189 (2007).

[11] H. Na and T. Endoh, Jpn. J. Appl. Phys. 52, 04CE08 (2013).
[12] D.-B. Kao, J. P. McVittie, W. D. Nix, and K. C. Saraswat, IEEE Trans. Electron Devices 34, 1008 (1987).

[13] D.-B. Kao, J. P. McVittie, W. D. Nix, and K. C. Saraswat, IEEE Trans. Electron Devices 35, 25 (1988).

[14] C. S. Rafferty and R. W. Dutton, Appl. Phys. Lett. 54, 1815 (1989).

[15] H. I. Liu, N. I. Maluf, and R. F. W. Pease: J. Vac. Sci. Technol. B Nanotechnol Microelectron 10, 2846 (1992).

[16] H. I. Liu, D. K. Biegelsen, N. M. Johnson, F. A. Ponce, and R. F. W. Pease, J. Vac. Sci. Technol. B Nanotechnol Microelectron 11, 2532 (1993).

[17] H. I. Liu, D. K. Biegelsen, F. A. Ponce, N. M. Johnson, and R. F. W. Pease, Appl. Phys. Lett. 64, 1383 (1994).

[18] C. C. Büttner and M. Zacharias, Appl. Phys. Lett. 89, 263106 (2006).

[19] D. Shir, B. Z. Liu, A. M. Mohammad, K.-K. Lew, and S. E. Mohney, J. Vac. Sci. Technol. B Nanotechnol Microelectron 24, 1333 (2006).

[20] H. Kageshima, K. Shiraishi, and T. Endoh, Jpn. J. Appl. Phys. 55, 08PE02 (2016).

[21] H. Kageshima, M. Uematsu, K. Akagi, S. Tsuneyuki, T. Akiyama, and K. Shiraishi, Jpn. J. Appl. Phys. 43, 8223 (2004).

[22] M. Uematsu, H. Kageshima, and K. Shiraishi, Jpn. J. Appl. Phys. 44, 7756 (2005).

[23] H. Kageshima, M. Uematsu, K. Akagi, S. Tsuneyuki, T. Akiyama, and K. Shiraishi, e-J. Surf. Sci. Nanotech. 4, 
584 (2006).

[24] H. Kageshima, M. Uematsu, K. Akagi, S. Tsuneyuki, T. Akiyama, and K. Shiraishi, Jpn. J. Appl. Phys. 45, 694 (2006).

[25] H. Kageshima, M. Uematsu, K. Akagi, S. Tsuneyuki, T. Akiyama, and K. Shiraishi, Physica B Condens. Matter 376-377, 407 (2006).

[26] M. Uematsu, H. Kageshima, S. Fukatsu, K. M. Itoh, K. Shiraishi, M. Otani, and A. Oshiyama, Thin Solid Films 508, 270 (2006).

[27] H. Omi, H. Kageshima, and M. Uematsu, Phys. Rev. Lett. 97, 016102 (2006).

[28] S. Hosoi, K. Nakajima, M. Suzuki, K. Kimura, Y. Shimizu, S. Fukatsu, K. M. Itoh, M. Uematsu, H. Kageshima, and K. Shiraishi, Nucl. Instrum. Methods Phys. Res. B 249, 390 (2006).

[29] H. Kageshima, M. Uematsu, T. Akiyama, and T. Ito, Jpn. J. Appl. Phys. 45, 7672 (2006).

[30] S. Izumi, T. Miyake, S. Sakai, and H. Ohta: Mater. Sci. Eng. A 395, 62 (2005).
[31] I. Yonenaga and K. Sumino, Jpn. J. Appl. Phys. 21, 47 (1982).

[32] I. Yonenaga, K. Sumino, and Kinji Hoshi, J. Appl. Phys. 56, 2346 (1984).

[33] J. R. Patel, K. A. Jackson, and H. Reiss, J. Appl. Phys. 48, 5279 (1977).

[34] K. Wada, H. Takaoka, N. Inoue, and K. Kohra, Jpn. J. Appl. Phys. 18, 1629 (1979).

[35] H. Ohta, and M. Kitano, J. Soc. Mat. Sci. 46, 1101 (1997).

[36] M. Aaktsuka, K. Sueoka, H. Katahama, N. Morimoto, and N. Adachi, Jpn. J. Appl. Phys. 36, L 1422 (1997).

[37] Model number: JEOL JEM-ARM200F Double Cs Corrector, cold FEG gun / Acceleration voltage: $200 \mathrm{kV}$, STEM-HAADF \& ABF-STEM / Magnification: 4M and 10M, Resolution: $0.1 \mathrm{~nm}$.

[38] H. Seki, TORAY Research Center, The TRC Journal, 1 (2015). 